\title{
Corporate Social Responsibility - Instrumente zur Gestaltung transnationaler Arbeitsbeziehungen
}

Im Rahmen von unternehmerischen Politiken zu „gesellschaftlicher Verantwortung“ (Corporate Social Responsibility, CSR) wächst der Stellenwert von Sozialstandards. Über soft law-Instrumente erheben CSR-Politiken auch Anspruch auf die Gestaltung der transnationalen Arbeitsbeziehungen. Das wirft die Frage auf, welche Rolle Gewerkschaften und Beschäftigtenvertreter als originäre Akteure der Arbeitsbeziehungen bei der Formulierung und Setzung privater transnationaler Sozialstandards wie auch bei deren Kontrolle spielen.

\section{CSR und Sozialstandards}

Corporate Social Responsibility (CSR): Das ist - nach einer Definition der Europäischen Kommission - „im Wesentlichen ein Konzept, wonach Unternehmen sich freiwillig dazu entscheiden, zu einer besseren Gesellschaft und einer saubereren Umwelt beizutragen" (Europäische Kommission 2001, S. 4f.; Europäische Kommission 2006; Bassen et al. 2006). Die starke Betonung der Freiwilligkeit ist einer der Gründe dafür, weshalb Corporate Social Responsibility in Kontinentaleuropa kaum als probates Mittel zur Regulierung von Arbeitsbeziehungen gesehen wird (HauserDitz/Wilke 2005; Sobczak 1996; Daugareilh 2005, S. 353f.). Für die Regulierung von Arbeitsbeziehungen stehen schließlich rechtliche Garantien und Kollektivverhandlungen bereit, und soft law-Regelungen dienen - in Konkurrenz zum Recht häufig als Mittel zur Deregulierung. Solche grundsätzlichen Bedenken gegen soft law sind für den Bereich des transnationalen Handelns aber von geringerer Bedeutung. Denn die völkerrechtlichen Konventionen der International Labour Organisation (ILO) binden ohnehin nur Staaten, nicht jedoch private Unternehmen. Damit unterliegt die private Unternehmenstätigkeit nur den Regeln des nationalen Rechts am Unternehmensstandort. Es gibt also bis heute keine rechtlich bindenden Regeln, die private Unternehmen unmittelbar auf die Einhaltung von transnationalen Mindestsozialstandards verpflichten würden (Servais 2005). Hier setzen CSR-Konzepte an.

\subsection{TRANSNATIONALISIERUNG INTERNATIONALER SOZIAL- STANDARDS}

Als Instrumente zur privaten Regulierung von Sozialstandards werden im CSR-Kontext verwendet:

(1) einseitige unternehmerische Erklärungen, Selbstverpflichtungserklärungen bzw. Verhaltenskodizes/Codes of Conduct und

(2) Internationale Rahmenabkommen (International Framework Agreements, IFAs), also Vereinbarungen von Unternehmen mit gewerkschaftlichen Akteuren (überwiegend globale Gewerkschaftsföderationen wie der Internationale Metallarbeiterbund (IMB) und/oder Betriebsräte (Europäische Betriebsräte oder Weltbetriebsräte)) (vgl. Brandl/Stelzl 2005; Greven 2006).

Diese soft law-Instrumente haben in erster Linie bestimmte international-rechtliche Standards zum Gegenstand. Vor allem neuere Instrumente (die etwa ab dem Jahr 2000 verabschiedet wurden) enthalten regelmäßig das Verbot von Kinderarbeit, das Verbot von Zwangsarbeit, das Gebot der Nicht-Diskriminierung sowie das Recht auf Vereinigungs- und Kollektivverhandlungsfreiheit. Dies sind nichts anderes als die Kernarbeitsnormen aus der ILOErklärung von 1998 (vgl. Hepple 2005, S. 56ff.; Europäische Kommission 2001). Rückblickend zeigt sich hier ein Erfolg der ILO-Erklärung über Kernarbeitsnormen; sie wurde unter anderem auch in der Überarbeitung der OECD-Guidelines im Jahre 2000 berücksichtigt, die wiederum eine wichtige Referenz für private Standardsetzung sind. Wo heute von trans- oder inter- nationalen Mindeststandards die Rede ist, stößt man so immer wieder auf die ILOKernarbeitsnormen. Weitere Inhalte der genannten soft law-Instrumente sind Vorschriften zum Arbeits- und Gesundheitsschutz, Mindestlohn, Urlaub und zur Qualifizierung von Beschäftigten.

Solche transnationalen Standards haben bislang die Arbeitsbedingungen vor Ort zwar allenfalls in geringem Ausmaß verändert (vgl. Köhnen 2002; Hepple 2005; zu den Hintergründen auch Fichter/Sydow 2002). Sie können aber zumindest einen Referenzpunkt für regelkonformes und davon abweichendes Verhalten setzen. Auch Normverstöße gehören zum Begriff der Norm (O’Rourke 2003, S. 1; Brown Weiss/ Jacobson 1998, S. 4). Für die Beurteilung der Effektivität eines normativen Standards ist deshalb die Autorität einer Norm mindestens so wichtig wie die empirische Normbefolgung. Und Autorität in einem normativen Sinn setzt die Installierung von funktionierenden Implementationsund Kontrollmechanismen voraus (Abbott et al. 2000).

\subsection{SPEZIFIZITÄT DER STANDARDS UND KONKRETISIERUNG}

Die Implementations- und Kontrollmechanismen sind noch aus einem weiteren Grund zentral für die Beurteilung der Ef-

Eva Kocher, Dr., Leiterin der Akademie der Arbeit in der Universität Frankfurt/Main. Arbeitsschwerpunkte: Kollektives Arbeitsrecht, transnationale Sozialstandards, Verfahrensrecht.

e-mail: kocher@em.uni-frankfurt.de 
fektivität: In den Codes of Conduct und auch in einigen IFAs werden die einschlägigen ILO-Konventionen oft nicht ausdrücklich genannt. Viele der Texte sind von hohem Allgemeinheitsgrad und konkretisieren zum Beispiel nicht, welches Mindestalter für Erwerbsarbeit maßgeblich sein soll (im Fall der Kinderarbeit) oder welche Höchstarbeitszeiten gelten sollen.

Außerdem zeichnen sich die Sozialstandards durch eine Vielfalt von Flexibilitätsklauseln und Vorbehalten aus. Die am weitesten verbreitete Klausel dürfte der Hinweis auf das jeweilige nationale Recht oder die nationale Praxis sein: „Das national geltende Arbeitsrecht ist einzuhalten ". Ausdrückliche Günstigkeitsklauseln, wonach jeweils der höchste Standard anwendbar sein soll, sind selten. Fragt man Unternehmensvertreter und -vertreterinnen, die für die Umsetzung solcher Standards zuständig sind, nach der Anwendung der Klausel in der Praxis, ${ }^{2}$ so erfährt man, dass die internationalen Sozialstandards letztlich unter Berücksichtigung der lokal und regional üblichen Standards konkretisiert würden. Die Unternehmen interpretieren „ihre“ Standards also unter Bezugnahme auf das nationale Recht vor Ort. Dieses dient dann nicht als Mindeststandard, sondern als Bezugspunkt und Mittel, um zu konkretisieren, welche Normen im jeweiligen Unternehmen gelten sollen (Daugareilh 2005, S. $371 \mathrm{ff}$.; S. 378ff.).

Dies hat zur Folge, dass man nur wenig über einen Unternehmensstandard und seine Umsetzung sagen kann, wenn man nicht weiß, in welchen Fällen, aus welchen Anlässen und unter welchen institutionellen Rahmenbedingungen er konkretisiert wird. Je vager und allgemeiner Sozialstandards formuliert sind, desto mehr kommt es darauf an, wie Umsetzung und Implementierung erfolgen und welche Akteure mit welchen Interessen beteiligt sind.

\subsection{INSTRUMENTE ZUR IMPLEMEN- TATION UND KONTROLLE}

Mittlerweile ist eine Vielzahl von Implementations- und Durchsetzungsinstrumenten entwickelt worden: Sie reichen von Training und Schulung der Zulieferunternehmen über Stichprobenkontrollen bis hin zu systematischem oder gar externem Monitoring und Auditing. Vorherrschend sind nach wie vor interne Implementationsmechanismen, wie die Steuerung der Personalpolitik in ausländischen Nieder- lassungen und abhängigen Unternehmen, Monitoring vor Ort, die Nutzung des Qualitäts- und Prozessmanagements (Stichproben anlässlich der Qualitätskontrolle) oder die Schulung von Zulieferern. Daneben sind in einigen Fällen externe Mechanismen, wie die Auditierung von Zulieferern und damit die Einbeziehung von unternehmensfremden Auditoren und Kontrolleuren, getreten (Überblick bei Hepple 2005, S. 75; Köpke/Röhr 2003). Zertifizierungsagenturen, die soziale Labels vergeben, tragen dazu bei, die Implementierung von Sozialstandards bzw. Monitoringverfahren allmählich zu vereinheitlichen, da sie eine gewisse Kontrolle von außen verlangen. In der Auditierung sind manchmal auch Nicht-Regierungsorganisationen (NGOs) als Auditoren beteiligt (ausführlicher Überblick: O'Rourke 2003, S. 11ff.). Die Entwicklung geht insgesamt dahin, nicht nur systematisch oder anlassbezogen zu kontrollieren, inwieweit die materiellen Arbeitsstandards vor Ort dem Wortlaut der vereinbarten Sozialstandards entsprechen, sondern auch Implementations- und Kontrollverfahren zu professionalisieren bzw. zu institutionalisieren. Auffallend ist in diesem Zusammenhang die Bedeutung von Managementsystemen und Mainstreaming-Mechanismen. Sie gelten mittlerweile als „state of the art“, um zu gewährleisten, dass Prinzipien sozialer Verantwortung in ökonomische Strategien eingebunden werden.

\section{Die Beteiligung von Arbeitnehmervertretungen}

\subsection{EINSEITIGE CODES OF CONDUCT}

Bei Selbstverpflichtungserklärungen von Unternehmen, transnationale Sozialstandards einzuhalten, behält das Unternehmen selbst die Kontrolle über die Implementation der Norm. Es bleibt Herr des Verfahrens, wenn es darum geht, die Norm(en) auszulegen und zu konkretisieren oder darüber zu entscheiden, ob externe Auditoren einbezogen werden. Eine wirkliche Delegation des Kontrollprozesses, wie er Voraussetzung einer „rechtlichen“ Autorität von Standards wäre (Abbott et al. 2000), findet nicht statt.

Bei der Umsetzung solcher einseitigen Codes of Conduct werden Interessenver- tretungen der Beschäftigten nur selten einbezogen. Dies gilt sowohl dort, wo lediglich internes Monitoring und Stichprobenkontrollen praktiziert werden, als auch dort, wo externe Auditierung und Überprüfungsverfahren stattfinden. Die Forderungen des Internationalen Gewerkschaftsbundes (IGB) nach einer effektiven Beteiligung von Gewerkschaften und Beschäftigtenvertretern beim Monitoring sind so noch weitgehend unerfüllt (Hepple 2005, S. 75).

\subsection{INTERNATIONAL FRAMEWORK AGREEMENTS}

Eine Ausnahme besteht jedoch im Falle der International Framework Agreements (IFAs), bei denen sowohl die Standards von Unternehmens- und Gewerkschaftsseite gemeinsam gesetzt werden als auch Implementations- und Kontrollverfahren zusammen entwickelt werden (Seifert 2006; Sobczak 2004; Brandl/Stelzl 2005; Greven 2006). Gerade neuere IFAs zeichnen sich dadurch aus, dass die Arbeitnehmervertretungen aktiv in den Implementationsprozess einbezogen sind. In der Regel werden gemeinsame Arbeitsgruppen eingerichtet oder regelmäßige Treffen, Beschwerdeoder Berichtsmechanismen verabredet. In Einzelfällen haben auf internationaler Konzernebene getroffene Vereinbarungen auch dazu geführt, dass Gewerkschaften vor Ort vom lokalen Management (erstmals) anerkannt werden bzw. dass sich betriebliche Repräsentationsstrukturen etablieren können (Baylos Grau 2006, S. 82ff.). Da die internationalen Gewerkschaftsorganisationen nur über begrenzte Ressourcen

\footnotetext{
So zum Beispiel der Code of Conduct der Otto Group (http://www.otto.com/Code of conduct.84.0.html); siehe auch VW-Sozialcharta vom 6.6.2002, Arbeit und Recht 2002, S. 343

2 Entsprechende Befragungen wurden im Rahmen des Projektes "Régulation Sociale des Entreprises Transnationales Européennes " zwischen 2004 und 2007 in sieben (west-)europäischen Staaten durchgeführt (vgl. http://ester.u-bordeaux4.fr). Empirische Basis für Deutschland sind 40 leitfadengestützte Interviews mit Vertretern aus vier Unternehmen und ihren Stakeholdern (Gewerkschaften, Betriebsräten, Verbraucherorganisationen, Menschenrechtsorganisationen/advokatorischen NGOs, Rating-Agenturen der Finanzmärkte, Investoren und Wirtschaftsvereinigungen). Das deutsche Team bestand aus Ulrich Zachert, Eva Kocher, Angelina Topan, Reingard Zimmer und Stefan Reuyß (Koordination Eva Kocher).
} 
verfügen, die Normeinhaltung zu überwachen, setzen IFAs ohnehin implizit voraus, dass Beschäftigte und lokale Gewerkschaften vor Ort sich aktiv an der Umsetzung und Einhaltung vereinbarter Standards beteiligen (Weinz 2006).

Der Grad der Formalisierung von Implementation und Kontrolle ist in diesen Fällen niedrig. Aussagen aus Interviews mit Experten (vgl. Fußnote 2) deuten darauf hin, dass dies damit zu tun haben mag, dass die International Framework Agreements Ausdruck kooperativer Arbeitsbeziehungen sind, in denen eine Überwachung von Arbeitsrechten oft eher informeller Art ist. So erläuterte ein betrieblicher Arbeitnehmervertreter, man verlasse sich vor allem auf „unsere Netzwerke auf Gewerkschaftsebene“, um Informationen über Verstöße zu erhalten.

\section{3 "BEST PRACTICE“ ODER "MAINSTREAM"}

Mit den International Framework Agreements sind neue Ansatzpunkte für Arbeitnehmerbeteiligung in transnationalen Arbeitsbeziehungen entwickelt worden. Sie setzen nicht zuletzt darauf, die CSR-Politik zu nutzen, um Räume für die Organisierung und die Stärkung der Akteure und Beschäftigten vor Ort zu schaffen.

Weltweit gesehen ist jedoch nur ein äußerst kleiner Teil der unternehmerischen Selbstverpflichtungen Ergebnis von Verhandlungen zwischen Unternehmens- und Gewerkschaftsseite. Und bei der Minderheit von Unternehmen wiederum, die das Instrument International Framework Agreement verwenden, zeigt sich: ${ }^{3} 49$ der 55 Unternehmen sind europäischen Ursprungs und haben ihren Hauptsitz in einem europäischen Land (Greven 2006, S. 12; Daugareilh 2005, S. 356); allein 37 sind deutsche, französische oder nordische Unternehmen, haben also einen (rechts-)kulturellen Hintergrund, der in besonderer Weise durch kooperative Arbeitsbeziehungen geprägt ist. Die größte Zahl an Vereinbarungen gibt es in der Metallindustrie (insgesamt 15). Neben der Tatsache, dass die Überwachung überwiegend über informelle gemeinsame Kommunikationsprozesse organisiert wird, deuten auch diese Daten darauf hin, dass es einen engen $\mathrm{Zu}$ sammenhang zwischen IFAs und einer Unternehmenskultur kooperativer Arbeitsbeziehungen bzw. dem Europäischen Sozialmodell gibt.
Dies wiederum wirft die Frage auf, ob IFAs dazu beitragen können, das Europäische Sozialmodell bzw. kooperative Arbeitsbeziehungen zu „exportieren“. Diese Chance scheint zu bestehen, wenn man in Rechnung stellt, dass die Verbreitung von International Framework Agreements ganz offensichtlich erst an ihrem Anfang steht: Alle (bis auf sechs) der vorgenannten Vereinbarungen wurden seit dem Jahr 2000 abgeschlossen; 26 Vereinbarungen gelten gar erst seit 2004. Für die Antwort auf die Frage, ob die Regelungsmodelle in den IFAs auch Maßstäbe für den Mainstream der CSR-Politiken setzen können, ist es jedoch wichtig zu klären, welche Sozialmodelle bzw. welche Vorstellungen von Arbeitsbeziehungen und Arbeitnehmerbeteiligung in diesem Mainstream vorherrschend sind.

\section{Typen von CSR-Politiken}

Bei Unternehmen, die transnationale Sozialstandards in ihre Unternehmenspolitik integrieren, lassen sich unterschiedliche Typen unterscheiden. Maßgeblich für die derzeitig feststellbaren Differenzen zwischen den Unternehmen ist, welche Akteure mit welchen Interessen die Anwendung und Durchsetzung der privaten Standards vorantreiben.

Um es vorweg zu nehmen: Die wichtigsten Akteure in der Anwendung und Kontrolle der Standards sind gegenwärtig die Standard setzenden Unternehmen selbst. Sie haben in aller Regel auch die Herrschaft über Implementations- und Kontrollinstrumente. Wenn also bei dem jetzigen Stand von einer Netzwerkkoordination (zum Begriff vgl. Börzel 1998) bei der Standardsetzung und -kontrolle zwar (noch) nicht die Rede sein kann, so haben transnationale Unternehmen dennoch den unterschiedlichsten Formen externen Drucks und externer Anforderungen gerecht zu werden. Im Einzelnen finden sich hier zwar je nach Unternehmen große Unterschiede. Generell lässt sich aber sagen, dass der Einfluss externer Akteure auch davon abhängt, inwieweit das Unternehmen selbst ein Interesse an Kooperation oder zumindest an Rücksichtnahme auf Stakeholder hat. Dies gibt externen Akteurinnen und Akteuren eine gewisse Macht, Erwartungen an Unternehmen zu formulieren und damit auf formelle oder auf informelle Weise direkt oder indirekt an der Definition, Konkretisierung, Anwendung und Kontrolle der Standards mitzuarbeiten.

Unternehmerische Interessen, die nichtunternehmerischen Akteurinnen und Akteuren Raum zur Einflussnahme und für eine Agenda-Setzung im Anwendungsprozess eröffnen, lassen sich wie folgt typisieren:

\section{1 ÖKONOMISCHE STRATEGIEN}

Einige der Vorreiter bei der Etablierung von Corporate Social Responsibility in Europa waren Unternehmen, die diese Politiken aus internen Gründen bzw. im Zusammenhang mit einer ökonomischen Strategie entwickelt haben. Eine Reihe von Unternehmen integriert CSR-Politiken in ihr Unternehmensselbstverständnis, in ihre corporate culture. Die „intrinsischen“ ökonomischen Interessen an CSR sind unterschiedlicher Art. Zum Teil spielen unternehmerische Interessen an einer bestimmten Qualität der Produkte, Abläufe oder Arbeitskräfte eine Rolle (Nayebpour/Koehn 2003); zum Teil nutzen Unternehmen die Sozialstandards als Hebel, um Kontrolle über ihre Zulieferkette ausüben zu können (Hepple 2005, S. 70 f.); zum Teil wird CSR als Instrument zur Erschließung neuer Märkte und zur Positionierung von Marken eingesetzt. Das letztgenannte Interesse hängt eng mit der corporate culture zusammen. Gerade in der Markenpolitik dient das nach außen kommunizierte Unternehmensselbstverständnis gerne auch als Wettbewerbsinstrument.

Das jeweils repräsentierte Sozialmodell hängt in diesen Fällen von der jeweiligen Unternehmenskultur und dem Unternehmensselbstverständnis $\mathrm{ab}$ - und natürlich von bisherigen Erfahrungen in den Arbeitsbeziehungen. International Framework Agreements sind nicht zufällig vor allem von Unternehmen einer bestimmten Unternehmenskultur und -struktur abgeschlossen worden (vgl. Abschnitt 2.3).

\footnotetext{
3 Siehe zum Beispiel die Übersicht des französischen Observatoire sur la Responsabilité Sociétale des Entreprises aus dem Jahr 2007; mittlerweile veraltet ist die Übersicht der damaligen International Confederation of Free Trade Unions (ICFTU) auf http://www.icftu.org/displaydocument.asp?!ndex $=991216332 \&$ Language $=E N$.
} 


\subsection{MANAGEMENT DES ÖFFENTLICHEN RUFS}

Andere Vorreiter in Deutschland haben CSR-Politiken bereits Ende der 1990er Jahre in ihr Unternehmenshandeln integriert - nach dem Start Anfang der 1990er Jahre in den USA. Prominente Beispiele aus der Textil- und Sportartikelindustrie zeigen, dass die Ausrichtung auf CSR vielfach als Reaktion auf vorangegangene Skandalisierungen durch NGOs erfolgte. In einer globalisierten Welt können Presse und Öffentlichkeit Ereignisse in irgendeinem Teil der Welt aufgreifen; weit entfernte Ereignisse können zu Risiken werden. Transnationale advokatorische Netzwerke (Keck/Sikkink 1998, S. 8ff.) sind häufig in der Lage, einzelne Ereignisse in ausländischen Niederlassungen oder bei Zulieferern über die Presse zu skandalisieren und so Druck auf Unternehmen auszuüben (Rodríguez-Garavito 2005, S. 64ff.).

Politischer Druck aus der Zivilgesellschaft und/oder den Medien kann Unternehmen also dazu bringen, durch gezielte Politiken Risiken für den öffentlichen Ruf begrenzen zu wollen (Management des öffentlichen Rufs). Gerade Markenunternehmen haben ein erhebliches Interesse daran, solche Risiken soweit wie möglich zu kontrollieren und negative öffentliche Aufmerksamkeit zu verhindern (Zadek 1998; Sobczak 2004, S. 28). Die CSR-Politik ist in solchen Fällen Teil des unternehmerischen Risiko-Managements. Dies wiederum gibt gesellschaftlichen Akteuren, die aus Sicht des Unternehmens diese Risiken repräsentieren und in gewisser Weise kontrollieren können, die Macht, CSR-Agenden für die Unternehmen zu setzen oder zumindest mitzubestimmen (Keck/Sikkink 1998).

Vorreiter, Benchmarks und Best Practices für die Implementation und Kontrolle von CSR-Politiken sind zu einem guten Teil in einer solchen Interessenkonstellation entwickelt worden. Denn die Aufstellung von Standards alleine verringert die Risiken für die Marke nicht; sie rückt sie im Gegenteil nur stärker in die öffentliche Aufmerksamkeit. Ein Unternehmen, das bewusstes Risikomanagement betreiben will, ist gezwungen, sich um ein möglichst effektives Umsetzungssystem zu bemühen. Die Interessenkonstellation hinter der CSR-Politik hat insofern auch Einfluss auf die Effektivität.

Und es sind vor allem transnationale advokatorische Netzwerke, die die entspre- chenden Risiken produzieren und sich so eine indirekte Einflussnahme eröffnen. In diesen Netzwerken wiederum spielen Menschenrechtsgruppen und NGOs, welche die globalisierungskritische Öffentlichkeit repräsentieren, in der Regel eine zentrale Rolle (Rodríguez-Garavito 2005; zur Bedeutung auch Müller et al. 2006). Unternehmen des Typs „Management des öffentlichen Rufs" pflegen zum Teil den Dialog mit Menschenrechtsgruppen und anderen NGOs oder beteiligen sich an Multistakeholder-Foren. ${ }^{4}$ Auch die Medien werden so als „Verstärker" der öffentlichen Meinung zu wichtigen Akteuren.

Es überrascht deshalb nicht, wenn in diesem Prozess Beschäftigtenvertreter allenfalls eine untergeordnete Rolle spielen. Implementierung, Kontrolle und Überwachung werden hier vor allem über Managementsysteme institutionalisiert, die Beschäftigten und Beschäftigtenvertretern in der Regel keine aktive Rolle zuweisen. Auch bei externen Auditoren genießt die Vereinigungsfreiheit keine Priorität (Musiolek 2006, S. 47ff.).

Entsprechend lag der Schwerpunkt der öffentlichen und zivilgesellschaftlichen Politik zu transnationalen Sozialstandards lange Zeit auf der Frage der Kinderarbeit, nicht aber in der Gewährleistung von Vereinigungs- und Kollektivverhandlungsfreiheit. Diese hat sich erst mit der impliziten Verbreitung der ILO-Kernarbeitsnormen langsam als Standard durchgesetzt (pessimistischer Seifert 2006, S. 218ff.). Auch europäische NGOs haben seither Vereinigungs- und Kollektivverhandlungsfreiheit in Weltmarktfabriken zu einem ihrer Themen gemacht. Diese neu entstandenen Solidaritäten (Sobczak 2004, S. 25ff; S. 45ff.) dürften die Gefahr einer nur selektiven Durchsetzung von Beschäftigtenrechten jedoch nicht ausreichend bannen (Hepple 2005, S. 20). So sind Verstöße gegen die Vereinigungs- oder Kollektivverhandlungsfreiheit nur sehr selten Anlass für eine wirksame öffentliche Skandalisierung gewesen, die Umsetzungsdruck aufbauen könnte.

\subsection{SOGEFFEKTE}

Noch weitaus problematischer im Hinblick auf die Beteiligung von Arbeitnehmervertretern ist jedoch eine Interessenkonstellation, die erst in den letzten Jahren wahrnehmbar geworden ist. Denn nicht alle Unternehmen sind in gleicher Weise durch öffentlichen Druck beeinflussbar. Wie das
Risiko, Gegenstand einer öffentlichen Skandalisierung zu werden, nicht für alle Unternehmen gleich groß ist, so ist auch die ökonomische Bedeutung des äußeren Drucks für die Unternehmen ungleich.

CSR-Politiken haben sich in den letzten Jahren dennoch unabhängig von Markenpolitiken und Skandalisierungsrisiken immer stärker ausgebreitet. Wettbewerbsmechanismen sind zu einer neuen Schubkraft geworden. CSR ist „en vogue“, Sogeffekte und Mitläufereffekte sind entstanden. Dies wird in den von uns geführten Interviews (vgl. Fußnote 2) überaus deutlich. Ein Interviewpartner nennt dies „Schafsherdenprinzip“; "das [CSR] ist etwas, was man heute einfach haben muss."

Die Financial Community und der Kapitalmarkt - Fondsgesellschaften, Analysten und Rating-Agenturen - scheinen am aktivsten und nachhaltigsten zu diesen Sogeffekten beizutragen. Der Nachhaltigkeitsmanager eines Unternehmens sagt: „Wir sind verantwortlich auch für die Beantwortung aller Rankings, die mit Nachhaltigkeitsaspekten zu tun haben, wir werden im Jahr mit 25 Rankings überzogen. ...25 Rankings, die dann wichtig sind für Finanzmarktanalysten oder für (...) Investment“. Ein anderer beschreibt: „Also da gibt's starke Anforderungen von außen, auch aus der Szene professioneller Rater und Ranker, und da wird man mit Fragen irgendwelcher Art (...) überschüttet. Man kann fast sagen, wöchentlich einer im Augenblick".

Aber auch Lieferanten und Abnehmer, insbesondere wenn diese selbst eine Marke zu verteidigen haben, verlangen von ihren Geschäftspartnern in Europa die Einhaltung bestimmter Sozialstandards. Die Interviews geben auch Hinweise auf Zusammenhänge zwischen der CSR-Konjunktur und der Globalisierung: Je mehr ein Unternehmen sich als global player positioniert und je globaler und vernetzter es arbeitet, desto stärker wird es von den unterschiedlichsten Seiten mit der scheinbar schon selbstverständlichen Anforderung konfrontiert, eine CSR-Politik (zu der transnationale Sozialstandards gehören) zu betreiben.

\footnotetext{
4 Z. B. Multistakeholder-Forum "Runder Tisch Verhaltenskodizes" (<http://www.coc-runder-tisch. de>), das von der Deutschen Gesellschaft für Technische Zusammenarbeit (GTZ) moderiert wird.
} 
Die Politik verstärkt solche Sogeffekte zurzeit erheblich. So will die Europäische Union mit ihrer neu formulierten Politik zu CSR sogar einen Schwerpunkt bei kleinen und mittleren Unternehmen setzen, die bisher noch kaum CSR-Praktiken entwickelt haben (Europäische Kommission 2006; Bundesregierung 2006).

\subsection{REFRAMING UND EFFEKTIVITÄT}

In der Fallgruppe „Sogeffekte“ wird mit CSR-Politiken also ebenfalls auf externe Anforderungen reagiert, die sich im Unternehmen intern als Risiko darstellen. Diese externen Anforderungen und Erwartungen werden jedoch nicht von zivilgesellschaftlichen, sondern von wirtschaftlichen Akteuren und Geschäftspartnern produziert. So haben zum Beispiel einige Standard setzende Unternehmen als Zulieferer globaler Unternehmen diesen Geschäftspartnern gegenüber eine Berichtspflicht, die sich aber nur auf einem niedrigen Niveau bewegt. Auch Organisationen, die Sponsoring-Verträge mit Sozialklauseln abschließen, begnügen sich vielfach mit der Existenz einer Vereinbarung über Sozialstandards, sie sehen sich jedoch nicht explizit in einer Kontrollfunktion. Auf die Frage, welche Art der Überprüfung man vornehme, wenn öffentlich (z. B. durch NGOs) konkrete Vorwürfe gegen einen Geschäftspartner erhoben würden, sagt ein solcher Interviewpartner: „Also das Unternehmen hat zum Thema CSR einen ziemlich großen Apparat, der viel weiter ist, als wir das sind. Und wenn die uns sagen, das ist eine unwahre Geschichte, und sie das auch belegen können, dann gehen wir davon aus, dass das so ist. Aber wir machen nicht die Polizei, die dann hingeht und Untersuchungen macht und so weiter, sondern wir beziehen uns auf das, was die Abteilung des Unternehmens uns mitteilt".

Ähnliches gilt für die Kontrolle durch die Finanzmärkte. Investment-Fonds oder Rating-Agenturen, die Unternehmen hinsichtlich ihrer Performance im sozialen und ökologischen Bereich bewerten, verwenden hierfür in erster Linie Berichte der Unternehmen selbst. Der praktisch bedeutsame Dow Jones Sustainability Index (DJSI) zum Beispiel verlangt von den Unternehmen die Beantwortung eines umfangreichen Fragebogens. Darüber hinaus führt das Wirtschaftsprüfungsunternehmen PriceWaterhouseCoopers (PWC) Interviews mit Verantwortlichen und über- prüft die Antworten der Unternehmen auf offensichtliche Widersprüche. Interessant ist insofern, dass die Unternehmen hier insbesondere über prozedurale Maßnahmen zur Implementierung von Standards berichten sollen; für die Teilnahme an einem entsprechenden Rating wird so indirekt die Einführung entsprechender Managementsysteme nahegelegt. Bei der Rating-Agentur Scoris ${ }^{5}$ spielen die Arbeitnehmervertreter als Informationsquelle eine gewisse Rolle. Als Informationsquellen werden von ihnen darüber hinaus Geschäftsberichte, die Medien, eine eigene Datenbank sowie die Berichte kritischer Gruppen verwendet. Zumindest mittelbar sind hier also auch die Akteure präsent, die den öffentlichen Ruf auf Verbrauchermärkten repräsentieren, wie Menschenrechtsgruppen und NGOs.

Ein Problem sind die Implementationskontrollen auf der Basis von Unternehmensberichten dennoch, und zwar deshalb, weil Unternehmen, die nur von Sogeffekten mitgezogen werden, nicht notwendig bewusst ein eigenes Interesse mit dem CSR-Standard verbinden werden. Die Anforderungen, an denen sie sich orientieren, sind die Erwartungen des Wettbewerbs und der Märkte an global player. Die geringen Anforderungen, die von dort für die Erfüllung dieser Erwartungen gestellt werden, lassen es zu, dass die CSR-Politik in diesen Fällen oft lediglich bislang praktizierte Politiken fortschreibt. Auch das wird in den von uns und unseren europäischen Partnerteams geführten Interviews sehr deutlich. Eine solche Form der CSR-Strategie ließe sich deshalb auch als re-labelling oder re-framing bezeichnen.

Für viele Unternehmen neu ist häufig dennoch, dass Managementsysteme erwartet bzw. vorausgesetzt werden, die es leisten, die Implementation und Kontrolle von CSR-Konzepten in institutionalisierter Verantwortung zu steuern. Dies gilt zunehmend als Benchmarkkriterium. Und es ist eines der Signale dafür, dass die private Standardsetzung - jedenfalls im Bereich der Sozialstandards - weniger in Richtung einer globalen Pluralisierung weist als vielmehr in Richtung eines Imports US-amerikanischer Modelle der Unternehmenssteuerung (Hepple 2005, S. 86; McCrudden 1999, S. 175; ausführlich Buckel 2003, S. 177ff.). In diesen Modellen gibt es jedoch keinen expliziten Ort für eine Beteiligung von Arbeitnehmervertretern. Zum Teil setzen sie im transnationalen Kontext eine Formalisierung von Kommunikationsstrukturen voraus, die im Bereich der Arbeitnehmerbeteiligung kaum Entsprechungen hat. Die aktuelle Entwicklung zur Verbreitung von CSR-Politiken geht an den Modellen vorbei, die in International Framework Agreements entwickelt worden sind.

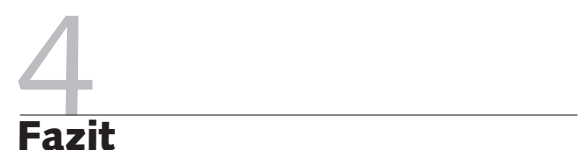

Fazit

Welche Interessenkonstellation - ökonomische Strategie, Management des öffentlichen Rufs, Sogeffekte - jeweils entscheidend für die CSR-Strategie eines Unternehmens ist, hängt von Branche und Größe, seinem Stellenwert als Markenanbieter sowie der Einbindung des Unternehmens in politische wie wirtschaftliche Netzwerke ab (Sobczak 2004). Hinter der Einführung transnationaler Sozialstandards steht oft eine Mischung unterschiedlicher Interessen. Allgemein wird der CSRMainstream jedoch zunehmend weniger von den „Leuchttürmen“ und „best practices" der CSR-Politik bestimmt, sondern immer mehr durch Unternehmen, die lediglich Sogeffekten der Finanzmärkte und globalen Wirtschaft folgen. Die hier maßgeblichen CSR-Stakeholder erwarten zwar auch die Einführung von Implementations- und Kontrollmechanismen; eine aktive Beteiligung der Beschäftigten und ihrer Interessenvertretungen hat in ihren Vorstellungen und Ansprüchen jedoch keinen Stellenwert. Die Standards, die in den International Framework Agreements entwickelt und gesetzt worden sind, verlieren in solchen Konstellationen ihren Vorbildcharakter. Damit sind Zweifel in zweierlei Richtung angebracht: zum einen, dass CSR-Konzepte an sich eine hinreichende Bedingung für die Gestaltung transnationaler Arbeitsbeziehungen sind; zum anderen, dass die Regelungsmodelle der IFAs den Mainstream beeinflussen können.

Hinzu kommt, dass gerade die vielfältigen informellen Funktionsmechanismen Kommunikation, Beschwerde und Infor-

\footnotetext{
5 Teil des Netzwerks SiRi (Sustainable Investment Research International Company Ltd., http:// www.siricompany.com).
} 
mationsaustausch -, die den IFAs zugrunde liegen, im Zuge der Globalisierung und Transnationalisierung eines Unternehmens an Grenzen stoßen. So funktioniert der interne Informationsaustausch unter den Arbeitnehmervertretern besser mit Beschäftigten in unternehmens- oder konzerneigenen ausländischen Niederlassungen als mit den Beschäftigten in Zulieferunternehmen. Auf Branchen, wie die Textilindustrie oder der Einzelhandel, in denen das transnationale Unternehmensgeschäft in komplexen Zulieferketten orga- nisiert ist (Fichter/Sydow 2002), sind die Mechanismen, die bislang in IFAs entwickelt wurden, noch nicht übertragen worden. In diesen Branchen bringt die Transnationalisierung jedoch gleichzeitig erhebliche Risiken für den öffentlichen Ruf von Marken mit sich. Diese Risiken haben zum Teil unternehmensexternen Akteuren, wie NGOs, Menschenrechtsgruppen und Medien, neue Einflussmöglichkeiten eröffnet, auf CSR-Standards zu dringen. Dass sie diese Chancen auch nutzen können, verdanken sie ihrer guten transnationalen
Vernetzung. Viele NGOs können auf die wichtige Ressource transnationaler Netzwerke zurückgreifen, bei den Gewerkschaften ist dies (noch) weit seltener der Fall (Hepple 2005, S. 76; Müller et al. 2006). Für die Zukunft der transnationalen Arbeitsbeziehungen wird es aber von Bedeutung sein, inwieweit es Gewerkschaften gelingt, sich auch in Interessenkonstellationen $\mathrm{zu}$ positionieren, in denen CSR-Konzepte durch ein „Management des öffentlichen Rufs" und durch "Sogeffekte" motiviert sind.

\section{LITERATUR}

Abbott, K. W./Keohane, R. O./Moravcsik, A./Slaughter, A.-M./Snidal,

D. (2000): The Concept of Legalization, in: International Organization 54, S. 401-419

Bassen, A./ Jastram, S./ Meyer, K. (2005): Corporate Social Responsibility. Eine Begriffserläuterung, in: Zeitschrift für Wirtschafts- und Unternehmensethik 2, S. 231-236

Baylos Grau, A. (2006): La responsabilidad legal de las empresas transnacionales, in: Revista de Derecho Social Latinoamérica 1, S. 69-99

Börzel, T. A. (1998): Organizing Babylon - on the Different Conceptions of Policy Networks, in: Public Administration 76, S. 253-273

Brandl, S./Stelzl, B. (2005): Internationale Arbeitsbeziehungen - Globalisierung als Chance für die deutschen Gewerkschaften?, in: WSI-Mitteilungen 2, S. 82-89

Brown Weiss, E./ Jacobson, H. (1998): A Framework for Analysis, in: Brown Weiss, E./ Jacobson, H. (Hrsg.): Engaging Countries: Strengthening Compliance with International Environmental Accords, S. $1 \mathrm{ff}$

Buckel, S. (2003): Empire oder Rechtspluralismus? Recht im Globalisierungsdiskurs, in: Kritische Justiz 2, S. 177-191

Bundesregierung (2006): Stellungnahme zur Mitteilung der Kommission „European Alliance“, vom 7.6.
Daugareilh, I. (2005): La responsabilité sociale des enterprises transnationales et les droits fondamentaux de I'homme au travail: Le contreexemple des accords internationaux, in: I. Daugareilh (Hrsg.): Mondialisation, travail et droits fondamentaux, Brüssel, S. 349-384

Europäische Kommission (2001): Grünbuch „Europäische Rahmenbedingungen für die soziale Verantwortung der Unternehmen“", KOM(2001)366 endg,

Europäische Kommission (2006): Mitteilung „Umsetzung der Partnerschaft für Wachstum und Beschäftigung: Europa soll auf dem Gebiet der sozialen Verantwortung der Unternehmen führend werden“", KOM(2006)136 endg.

Fichter, M./Sydow, J. (2002): Using Networks Towards Global Labor Standards? Organizing Social Responsibility in Global Production Chains, in: Industrielle Beziehungen 9, S. 357-380

Greven, T. (2006): Auf dem Prüfstand: Gewerkschaftsstrategien zur Regulierung globaler Konkurrenz, in: WSI-Mitteilungen 1, S. 10-15 Hauser-Ditz, A./Wilke, P. (2005), Corporate Social Responsibility Soziale und ökologische Verantwortung von Unternehmen. Eine Betriebsrätebefragung zu den Handlungsfeldern für Arbeitnehmervertretungen, www.boeckler.de/pdf/mbf_csr_diskussionspapier.pdf 
Hepple, B. (2005): Labour Laws and Global Trade, Oxford; Portland, Oregon

Keck, M./Sikkink, K. (1998): Activists beyond borders: advocacy networks in international politics, Ithaca, N. Y.

Köhnen, H. (2002): Haben Menschenrechtsverletzungen ein System? Wal-Mart's Verhaltenskodex und die Realität bei Zulieferern in ausge wählten Ländern, Düsseldorf: Hans-Böckler-Stiftung

Köpke, R./Röhr, W. (2003): Codes of Conduct. Verhaltensnormen für Unternehmen und ihre Überwachung, Hamburg

McCrudden, C. (1999): Human Rights Codes for Transnational Corporations: What Can the Sullivan and MacBride Principles Tell Us?, in: Oxford Journal of Legal Studies 19, S. 167-201

Müller, T./Platzer, H.-W./Rüb, S. (2006): Weltbetriebsräte und globale Netzwerke - Instrumente internationaler Solidarität?, in: WSI-Mitteilungen 1, S. 5-9

Musiolek, B. (2006): Interessenvertretungen in der Bekleidungsindustrie Mittel- und Südosteuropas, WSI-Mitteilungen 1, S. 47-52

Nayebpour, M. R./Koehn, D. (2003): The Ethics of Quality: Problems and Preconditions, Journal of Business Ethics (JOBE) 44, S. 37-48

O'Rourke, D. (2003): Outsourcing Regulation: Analysing Nongovernmental Systems of Labor Standards and Monitoring, in: The Policy Studies Journal 31, S. $1 \mathrm{ff}$
Rodríguez-Garavito, C. A. (2005): Nike's law: the anti-sweatshop movement, transnational corporations, and the struggle over international labor rights in the Americas, in: de Sousa Santos, B./Rodríguez-Garavito, C. A. (Hrsg.): Law and Globalization from below. Towards a Cosmopolitan Legality, Cambridge, S. 64ff

Seifert, A. (2006): Die Schaffung transnationaler Arbeitnehmervertretungen in weltweit tätigen Unternehmen, in: Zeitschrift für ausländisches und internationales Arbeits- und Sozialrecht 3, S. 205-224

Servais, J.-M. (2005), International Labour Law, Leiden

Sobczak, A. (2004): La responsabilité sociale de l'entreprise, menace ou opportunité pour le droit du travail?, in: Relations Industrielles - Industrial Relations 59, S. 26-51

Ulbrich, M. (2004): Enforcing Core Labour Rights through the OECDGuidelines for Multinational Enterprises? Reflections on the Guidelines' Conciliations Process and the Current Linkage Discussion, in: Zeitschrift für Ausländisches und Internationales Arbeits- und Sozialrecht 4, S. 366384

Weinz, W. (2006): Globale Rahmenvereinbarungen zwischen Gewerkschaften und Konzernen, in: J. Martens (Hrsg.): Verbindliche Regeln für Multis - Corporate Accountability - Zwischenbilanz und Zukunftsperspektiven, Bonn u.a., S. 22-25

Zadek, S. (1998): Balancing Performance, Ethics and Accountability, in: Journal of Business Ethics 17, S. 1421-1441 